专家推介: 本文提出了一种基于泊松分布的日盲紫外电晕检测方法。该方法对紫外图像序列根据暗噪声统计模 型进行有无信号的判断, 然后利用所建立的紫外电晕目标检测模型得到紫外图像的泊松概率映射图, 最后基于 改进的最大类间方差法（Otsu）实现紫外电晕目标检测与提取。该方法结构简单，检测精度高，且性能鲁棒。

\title{
基于泊松分布的日盲紫外电晕检测
}

\begin{abstract}
闵超波 ${ }^{1}$, 顾 燕 ${ }^{2}$, 杨 锋 ${ }^{2}$
（1. 河海大学常州校区 物联网工程学院，江苏 常州 213000；2. 北方夜视技术股份有限公司，江苏 南京 211102）

摘要: 针对日盲紫外电晕探测噪声大、信号微弱等特点, 本文提出了一种利用泊松分布的日盲紫外电 晕检测方法。为了表征紫外电晕信号的时空域特性, 根据光电探测原理, 构建了基于泊松分布的紫外 电晕目标检测模型。针对紫外图像序列, 首先根据暗噪声统计模型进行有无信号的判断, 然后利用所 建立的紫外电军目标检测模型得到紫外图像的泊松概率映射图，最后基于改进的最大类间方差法 （Otsu）实现紫外电晕目标检测与提取。本文方法在多组紫外图像序列中进行测试与对比, 实验证明 该方法可以准确地检测出紫外图像中电晕目标, 方法结构简单, 检测精度高, 且性能鲁棒。
\end{abstract}

关键词: 日盲紫外; 电晕探测; 目标检测; 泊松分布

中图分类号：TP391 文献标识码：A 文章编号：1001-8891(2020)08-0715-07

\section{Corona Detection of Solar-Blind Ultraviolet via Poisson Distribution}

\author{
MIN Chaobo ${ }^{1}$, GU Yan ${ }^{2}$, YANG Feng ${ }^{2}$ \\ (1. College of Internet of Things Engineering, Hohai University, Changzhou 213000, China; \\ 2. North Night Vision Technology Corp., Ltd., Nanjing 211102, China)
}

\begin{abstract}
A novel model is proposed for the object detection of solar-blind ultraviolet corona using the Poisson distribution and based on the photoelectric detection theory. This model can be used to represent the spatiotemporal characterization of solar-blind ultraviolet corona. In ultraviolet image sequences, initially, the existence of ultraviolet corona is determined by the statistical model of dark noise. Subsequently, the Poisson distribution map of the ultraviolet image is obtained using the proposed model. Finally, an improved Otsu's method is applied to detect and extract the object of ultraviolet corona. The proposed method is successfully tested over many ultraviolet image sequences and compared with the other two methods. The experiment results demonstrate that the proposed method performs better in object detection and has higher accuracy in ultraviolet image sequences.
\end{abstract}

Key words: solar-blind ultraviolet, corona detection, object detection, Poisson distribution

\section{0 引言}

随着日盲紫外成像探测器的不断发展, 其在电力 系统检测中的应用价值日益显著。与红外成像检测、 脉冲电流检测、声学检测以及紫外脉冲检测等传统检 测手段 ${ }^{[1-2]}$ 相比, 由于具备对电晕放电探测高灵敏度与 定位准确的特点, 使日盲紫外成像探测器有着不可替
代的优势 ${ }^{[3]}$ 。

由于 “日盲” 特性, 日盲紫外成像探测器可以得 到非常显著的电晕信号, 以及理想干净的背景信息, 且不受照度、风沙和雨雪等环境因素的影响。这一特 点非常有利于在日盲紫外图像中自动搜索电军放电 点, 实现对电晕放电目标的全自动搜索与跟踪。目前 电力系统检测主要依靠人工检测, 但是利用机器来代 
替人力进行检测是发展的必然趋势 ${ }^{[4]}$ 。因此, 开展日 盲紫外目标检测方法的研究, 可以推动电力系统自动 检测的实现。

紫外图像中快速准确地检测出目标是一项具有 挑战性的任务。由于紫外散射较大, 导致紫外电晕信 号在图像中分布离散, 而且放电现象本身是以脉冲的 形式出现 ${ }^{[5]}$, 使得紫外电晕信号时有时无, 无规律闪 炼, 再加上紫外探测器还会受到来自器件本身的随机 噪声和暗噪声的干扰 ${ }^{[6]}$, 因此在放电现象不明显的情 况, 紫外序列图像中包含了一堆分布离散、不间断闪 烁且位置不断变化的亮点, 很难确定紫外电晕目标的 准确位置, 原始成像效果如图 1 所示。
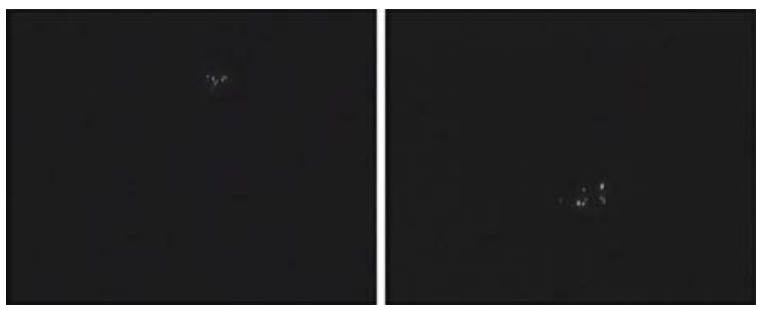

图 1 日盲紫外电晕成像效果示例

Fig. 1 The images of solar-blind ultraviolet corona

如上所述, 紫外电晕目标检测较为困难, 但其重 要性又非常明显, 因此近年来对紫外电晕目标检测的 研究也越来越多。文献[7]和[8]中介绍了一种利用形态 学的电晕信号检测方法, 利用阈值及腐蚀的方法来进 行紫外电晕目标的检测, 这种方法在放电量小、信号 分布离散的情况下效果退化严重。文献[9]提出了一种 基于 $\mathrm{MN}$ 规则的紫外电晕目标检测方法, 利用多帧累 积与简单的阈值法来进行紫外电晕目标检测, 虽然目 标检测一致性稍好, 但是检测效果对参数敏感, 容易 出现漏检现象。

针对日盲紫外电晕探测信号分布离散、无规律闪 炼跳跃的缺点, 本文提出了一种利用泊松分布的日盲 紫外电晕目标检测方法。首先, 利用泊松分布对紫外 电晕目标检测进行时空域建模。其次, 利用所建立的 紫外电晕目标检测模型, 得到存在电晕目标的紫外图 像泊松概率映射图。最后, 利用改进的最大类间方差 法（Otsu）实现紫外电晕目标检测与提取。本文方法 在多组紫外图像序列中进行测试与对比, 实验证明该 方法可以准确地自动检测出紫外图像中的电晕目标, 检测精度高, 且性能鲁棒, 可以提高日盲紫外电晕探 测的可靠性。

\section{1 基于泊松分布的日盲紫外电晕检测模型}

\section{1 日盲紫外成像探测模组组成原理}

日盲紫外成像探测模组基本构成如图 2 所示。电 晕所发出的紫外光信号通过紫外镜头与滤光片聚焦 于紫外 ICCD 入射窗上，从入射窗入射的光子照射到 光电阴极上, 按一定的量子转换效率转化为光电子, 在加速电场的作用下光电子进入微通道板 （microchannel plate, MCP）进行倍增, 然后聚焦到苂 光屏激发出可见光, 通过光纤光雉将图像耦合到可见 光 CCD 上, 最后由读出电路读出, 并且编码成数字 信号输出, 完成从入射光到数字图像的转换。

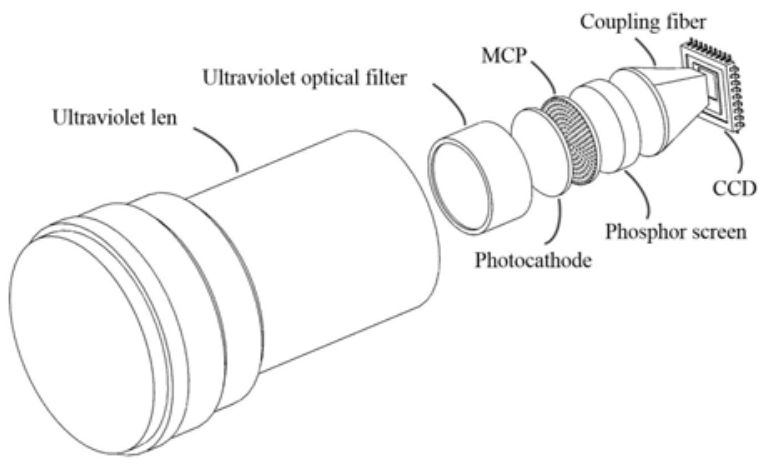

图 2 日盲紫外成像探测模组基本构成

Fig.2 The framework of solar-blind ultraviolet imaging module

\section{2 紫外电晕检测建模}

紫外电晕信号的探测是一种光电探测过程。对于 光电探测过程来说, 无论对光子流或光生电荷取样和 观察, 这一过程符合泊松分布 ${ }^{[10]}$ 。因此这一统计规律 同样适用于日盲紫外电晕信号的探测过程。

泊松分布定义如下：如果 $k \geqslant 0$ 个随机事件以已 知平均速率 $N / T$ 出现, 并与最后事件出现的时间无关, 那么在固定观察时间 $T$ 中观察到这些事件的概率 $p_{N}(k)$ 如下:

$$
p_{N}(k)=\frac{N^{k} \mathrm{e}^{-N}}{k !}
$$

式中: $N$ 表示在 $T$ 时间段中随机事件出现的平均个数; $k$ 表示观察到的随机事件的个数。

根据光电探测符合泊松分布这一规律, 可以假 设: 在 $m \times n$ 大小的序列紫外图像 $I$ 中,任意像素 $I(x, y)$ 探测到紫外电晕信号的概率都是符合泊松分布的, 其 中 $(x, y)$ 表示像素坐标。

因此, 任意像素 $I(x, y)$ 探测到紫外电晕信号概率 模型 $P(x, y)$ 可以表示为如下式:

$$
P(x, y)=1-p_{N}(0)=1-\mathrm{e}^{-N(x, y)}
$$

式中：均值映射图 $N(x, y)$ 表示在包含 $T$ 帧的紫外序列 图像中像素 $(x, y)$ 上出现紫外信号的平均个数, 可以表 示成如下公式: 


$$
N(x, y)=\frac{1}{T} \sum_{t=1}^{T} B^{t}(x, y)
$$

式中: $B^{t}$ 表示包含 $T$ 帧图像的紫外图像序列中第 $t$ 帧紫 外图像 $I$ 的二值化图像, 0 表示背景, 1 表示紫外信号, $T$ 也可称为积分时间。利用适当的阈值并配合传统 Otsu 算法来实现紫外图像的二值化。在本文中二值化阈值 取值为 50 , 且针对所有实验该参数都没有改变。

利用公式(2)和公式(3)对图像中所有像素进行遍 历之后, 就形成了图像序列在积分时间为 $T$ 情况下所 对应的均值映射图 $N$ 和基于泊松分布的概率映射图 $P$ 。概率映射图 $P$ 表征了在紫外信号探测过程中每个 像素不为 0 的概率, 即出现紫外电晕信号的概率, 反 映了紫外电晕目标时域特征在空域中的分布情况。

\section{2 日盲紫外电晕检测算法}

日盲紫外电晕目标检测算法总体流程如图 3 所 示, 主要由 3 部分组成。首先, 利用暗噪声统计模型 来判断当前帧紫外二值化图像是否有电晕信号。其 次, 如果存在信号则利用 1.2 节中的基于泊松分布的 概率模型计算当前帧的概率映射图 $P$ 。最后在概率映 射图 $P$ 中, 利用改进的 Otsu 算法提取电晕放电信号, 并输出电晕检测结果。此过程不断迭代, 就可以实现 对紫外序列图像的流水线处理。

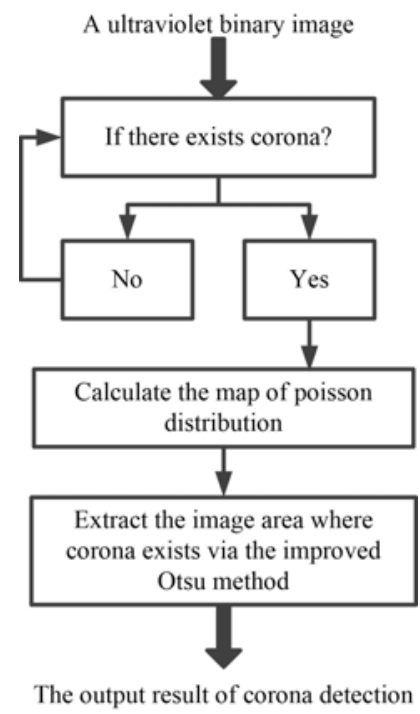

图 3 日盲紫外电晕检测算法总体流程

Fig. 3 The overview of our proposed algorithm

\section{1 暗噪声概率统计模型}

日盲紫外成像探测模组由于阴极、MCP 等因素的 影响, 所产生的暗噪声会造成电晕目标的虚警。但是 对于探测模组来说, 每帧图像中暗噪声的出现概率是 符合正态分布的, 因此可以利用暗噪声统计模型, 来
进行暗噪声与电晕信号的区分。根据参数估计的 $3 \sigma$ 准则, 有无电晕信号的阈值 $G$ 可以表示成如下公式:

$$
G=\mu_{\text {dark }}+3 \sigma_{\text {dark }}
$$

式中: $\mu_{\mathrm{dark}}$ 为暗噪声出现频率的平均值; $\sigma_{\mathrm{dark}}$ 为对应 的标准差, 这两个参数都需要对同一台日盲紫外相机 的大量暗噪声序列图像进行统计而得, 本文中选用 91236 帧暗噪声序列图像进行统计, 计算出相应的 $\mu_{\text {dark }}$ 和 $\sigma_{\text {dark }}$ 。

在实际使用中, 需要对每一帧输入的紫外图像进 行信号出现概率的计算, 若信号出现概率大于 $G$, 则 认为图像中存在电晕信号, 反之则无信号。

\section{2 泊松分布概率映射图 $\boldsymbol{P}$ 的计算}

根据公式(2)的泊松概率模型, 设计了一种快速计 算方法, 具体步骤如算法 1（迭代计算泊松分布概率 映射图) 所示。

输入: 紫外图像 $I$ 的二值化图像 $B$, 参数 $T$;

输出：泊松分布概率映射图 $P$;

将 $N$ 初始化为与 $B$ 大小一致的零矩阵, 并令 $t=0$; while 1

$$
\begin{aligned}
& t=t+1 ; \quad B^{t}=B ; \quad N=N+B^{t} \\
& \text { if } t=T \\
& N=N / T \\
& \text { for each pixel }(x, y) \text { in } N \\
& P(x, y)=1-\mathrm{e}^{-N(x, y)} ; \\
& \text { end for } \\
& \text { set } t=0 ; \\
& \text { end if } \\
& \text { return } P \\
& \text { end while }
\end{aligned}
$$

通过算法 1 的迭代, 根据实时输入的序列图像不 断更新均值映射图 $N$, 从而每隔 $T$ 帧更新泊松分布概 率映射图 $P$, 检测出的紫外电晕目标由离散渐渐聚集 为突出的光斑, 同时将泊松分布概率映射图 $P$ 进行归 一化处理, 就可以显示出当前紫外电晕信号空间分布 的情况。

\section{3 紫外电晕目标的提取}

由于泊松分布概率映射图 $P$ 中真正电晕目标像素 点比例低, 且相应的泊松概率值分布较窄, 因此利用 传统的 Otsu 法很难提取出准确的紫外电晕目标。为 了解决这一问题, 本文对传统的 Otsu 法进行了改进, 在进行直方图统计时增加了两步处理:

1）只对泊松分布概率映射图中概率大于 0 的像 素进行统计, 得到泊松分布概率直方图数组 $H$;

2) 对于 $H$ 中任意元素 $H(i)$, 如果 $H(i)=\max (\mathrm{H})$, 则将 $H(i)=0$ 。 
接下来利用 $H$ 再进行最大类间差的计算, 自动找 出最佳阈值, 就可以得到较为理想的目标提取结果。

\section{3 实验与分析}

\section{1 实验设备}

本文所使用的放电设备为充气型高压试验变压 器, 实物图如图 4 所示。主要由变压器、滤波器、限 流保护电阻、耦合电容、放电绝缘端子以及局放检测 仪组成。输入电压 $200 \mathrm{~V}$, 输出电压最高可达 $100 \mathrm{kV}$, 额定电压下局放量 $\leqslant 3 \mathrm{pC}$ 。

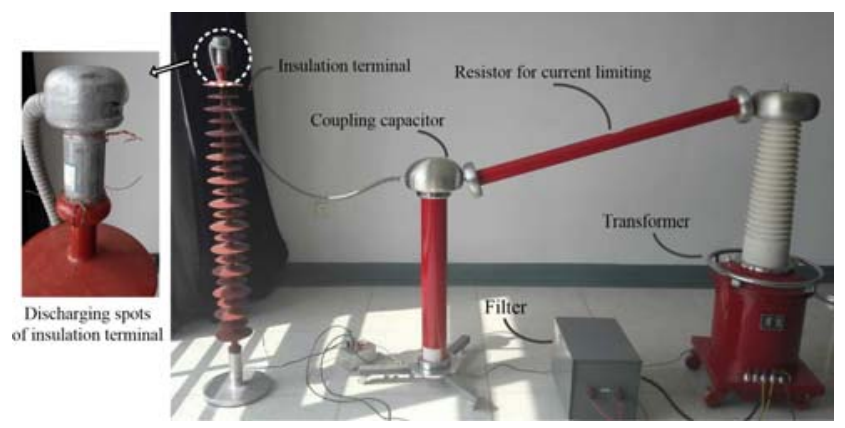

图 4 放电设备实物图

Fig. 4 The discharge equipment

在绝缘端子顶端加上铜丝, 加压后铜丝尖端就容 易形成位置固定且放电强度较稳定的电军放电点, 便 于日盲紫外电晕信号检测的研究。选用北方夜视技术 股份有限公司自主研发的数字化日盲紫外成像探测 模组进行紫外电晕信号探测, 实物如图 5 所示。

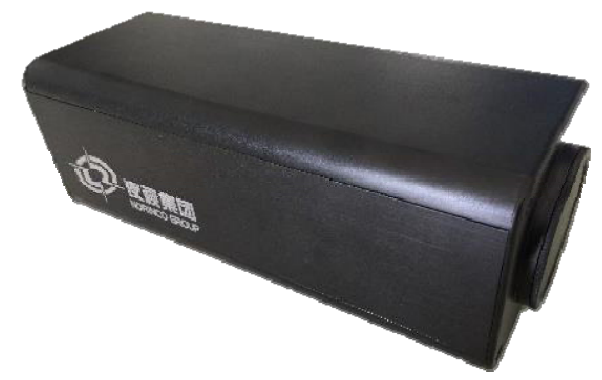

图 5 北方夜视数字化日盲紫外成像探测模组

Fig. 5 The solar-blind ultraviolet imaging module from NNVT

该模组采用 $\mathrm{TeCs}_{2}$ 光阴极紫外像增强器, 以透紫 外石英玻璃为输入窗的双近贴聚焦式像增强器, 配备 深截止日盲紫外滤光片、112 mm 焦距的紫外镜头以 及电子信号处理系统, 实现了全日盲波段的紫外信号 探测。

\section{2 评价指标}

为了测试本文方法的性能, 将其与文献[7]中介绍 的一种利用形态学的电晕信号检测方法进行比较。为 了客观定量地衡量两种方法的性能, 定义了两种指标 分别来衡量电晕目标检测的稳定性与准确性。
第一种为目标质心坐标标准差 $S$, 可以表示为如 下公式:

$$
S=\operatorname{std}\left(X_{c}\right)+\operatorname{std}\left(Y_{c}\right)
$$

式中: $X_{\mathrm{c}}$ 表示紫外序列图像中检测结果目标质心的横 坐标数组; $Y_{\mathrm{c}}$ 表示紫外序列图像中检测结果目标质心 的纵坐标数组; $\operatorname{std}(\cdot)$ 表示标准差运算。质心坐标标准 差可以反映图像序列检测结果的离散程度, 因此目标 质心坐标标准差 $S$ 越小, 说明检测性能越稳定。

第二种为目标质心检测精度 $A$ 定义如下:

$$
A=n_{\mathrm{c}} / n_{\mathrm{v}}
$$

式中: $n_{\mathrm{c}}$ 表示在原始紫外图像的二值化图像 $B$, 检测 结果目标质心位置的对应像素值为 1 (即有紫外信号) 的图像总帧数; 测试用的紫外图像序列总帧数。质心 检测精度直接反映了检测结果是否是真实的紫外电 晕信号, 可以用来表征检测方法检测的精度，质心检 测精度 $A$ 越高, 则说明检测越准确。

\section{3 单电晕目标检测的实验}

本文利用放电电压为 $8.5 \mathrm{kV} 、 19.5 \mathrm{kV}$ 和 $28 \mathrm{kV}$ 情 况下, 紫外模组增益电压为 $1.5 \mathrm{~V} 、 2.5 \mathrm{~V}$ 以及 $4 \mathrm{~V}$ 时 紫外模组对放电设备所拍摄的紫外序列图像进行实 验。在实验之前调整绝缘端子顶部铜丝形状, 使其只 有一个尖端放电点。实验用的紫外图像序列的图像大 小 $720 \times 576$, 帧数约为 3000 帧。

图 6 显示了本文方法在不同放电电压、不同增益 电压下最终的归一化泊松分布概率映射图以及紫外 电晕信号的提取结果。实验结果表明, 在多种不同条 件下, 本文提出的方法可以准确检测并提取出紫外电 晕目标，且没有虚警的情况发生。

图 7 显示了两种方法在不同放电电压、增益电压 情况下的质心坐标标准差计算结果，图 8 为质心检测 精度的计算结果。从中可以明显看出, 本文提出的方 法的质心坐标标准差和质心检测精度计算结果都大 大优于基于形态学的检测方法, 平均质心坐标标准差 仅为基于形态学检测方法的 10\%左右，而平均质心检 测精度却是基于形态学检测方法的 2.56 倍, 这充分说 明了本文提出的方法检测精度高, 紫外电晕目标定位 准确, 且效果鲁棒, 检测一致性好。

\section{4 标电晕检测的实验}

调整绝缘端子顶部铜丝形状, 使其产生 2 个尖端、 5 个尖端以及 6 个尖端 3 种放电情况, 将放电电压调 节至 $50 \mathrm{kV}$ ，紫外模组增益调节至 $3.5 \mathrm{~V}$, 对 3 种情况 进行探测并记录图像序列。文献[9]提出了一种基于 MN 规则的紫外电晕目标检测方法, 其本质为利用信 号频率模型来进行紫外电晕信号的检测。将其与本文 
提出的方法进行对比, 以进一步测试本文所提出的泊 松分布概率模型的性能。

图 9 分别显示了 5 个、 6 个和 2 个放电点情况下 的电晕检测结果。从中可以看出, 信号频率模型的结

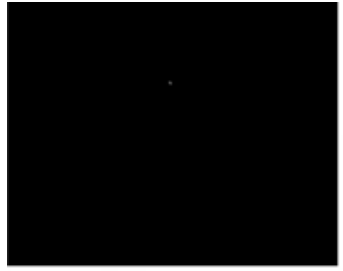

$28 \mathrm{kV}$

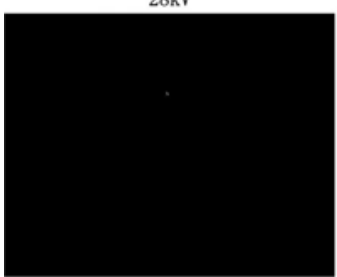

19. $5 \mathrm{kV}$

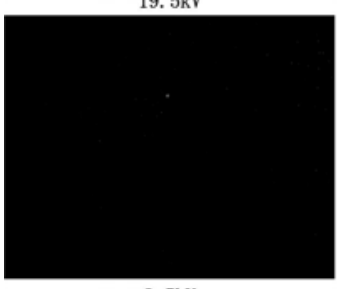

8. $5 \mathrm{kV}$
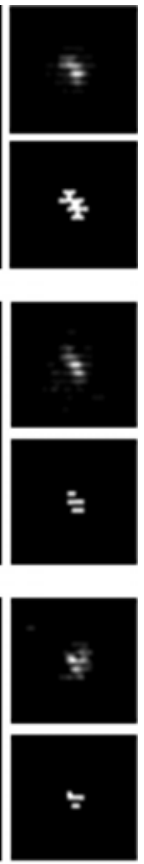

(a) Gain=1.5 V

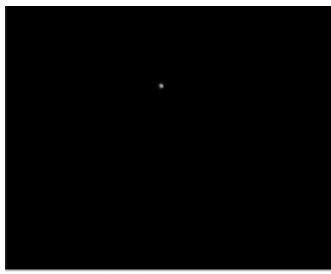

$28 \mathrm{kV}$

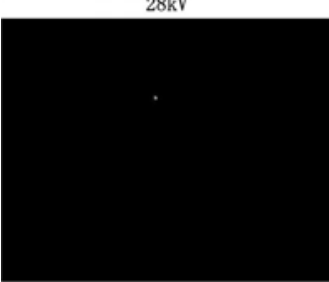

19. $5 \mathrm{kV}$

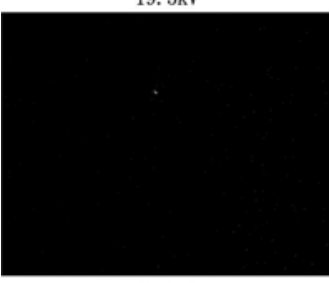

8. $5 \mathrm{kV}$

(b) Gain $=2.5 \mathrm{~V}$
果无法准确检测出所有的放电点电晕信号。并且相比 于本文方法, 基于信号频率的检测结果比较微弱, 不 够明显。因此对于多点放电情况下的目标检测, 本文 提出的方法检测精度更高，检测出的目标更加显著。
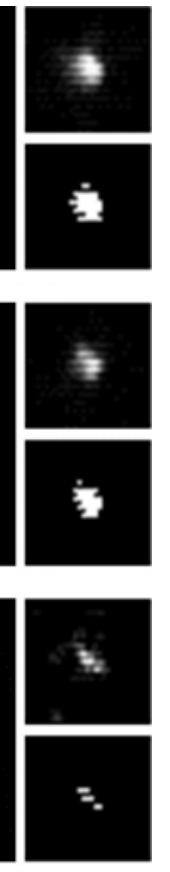

$=$
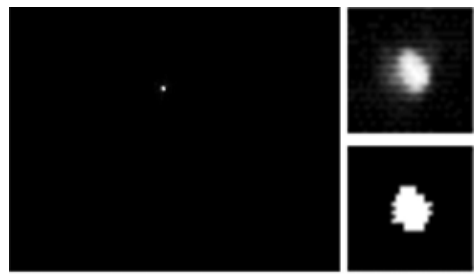

$28 \mathrm{kV}$
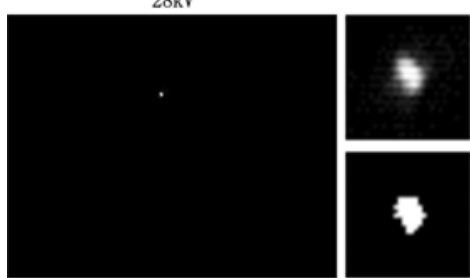

19. $5 \mathrm{kV}$
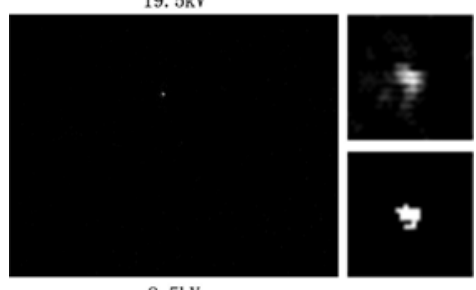

(c) Gain $=4 \mathrm{~V}$

图 6 本文提出方法在不同放电电压、不同增益电压下的电晕检测结果, 其中每一个放电电压下的检测结果图像组中, 左边为归一 化的泊松分布概率映射图, 右上角为检测到的电军目标的放大图, 右下角为电军信号目标提取结果的放大图

Fig. 6 The results of the proposed method with the different discharge voltage and gain, where the image on the left is the Poisson distribution map, the image on the upper right is the enlargement of object detection of ultraviolet corona, and the image on the low right is the enlargement of the extracting result

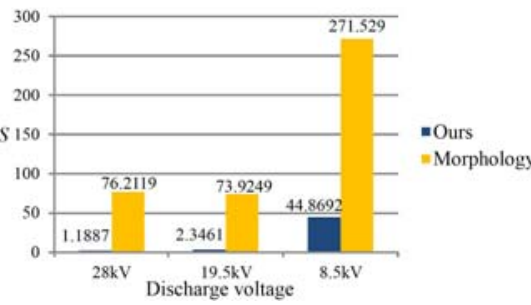

(a) Gain=1.5 V

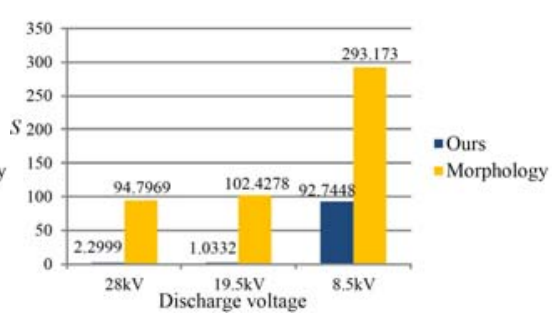

(b) Gain=2.5 V

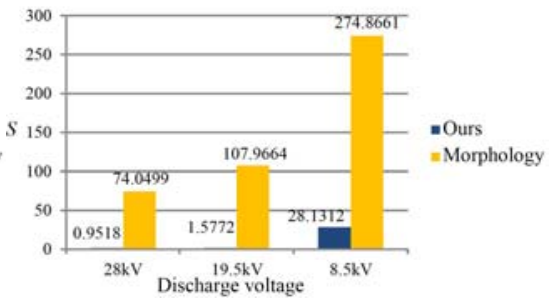

(c) Gain= 4 V

图 7 本文提出方法与形态学方法电晕检测的目标质心坐标标准差结果

Fig. 7 The standard deviation of object centroid of the proposed method and the morphology-based method

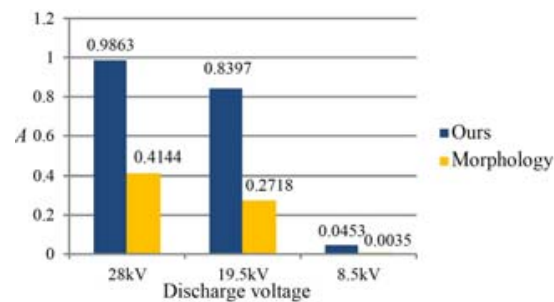

(a) Gain=1.5 V

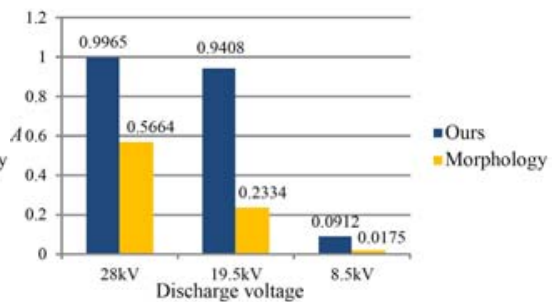

(b) Gain=2.5 V

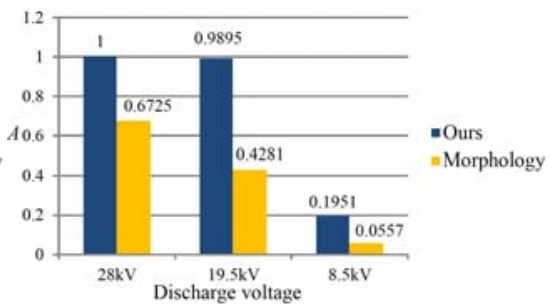

(c) Gain=4 V

图 8 本文提出方法与形态学方法电晕检测的目标质心检测精度结果

Fig. 8 The precision of object centroid of the proposed method and the morphology-based method 


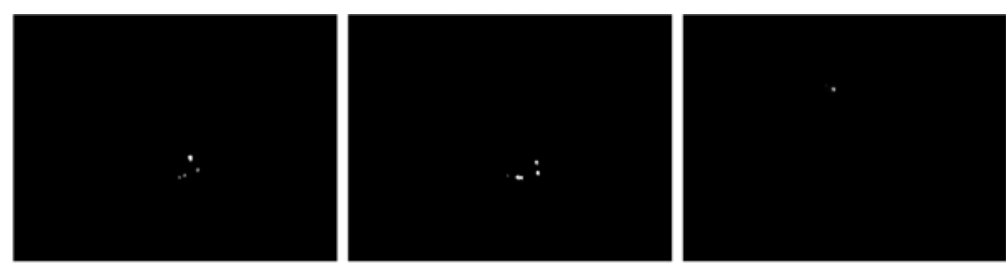

The results by the signal-frequency-based model
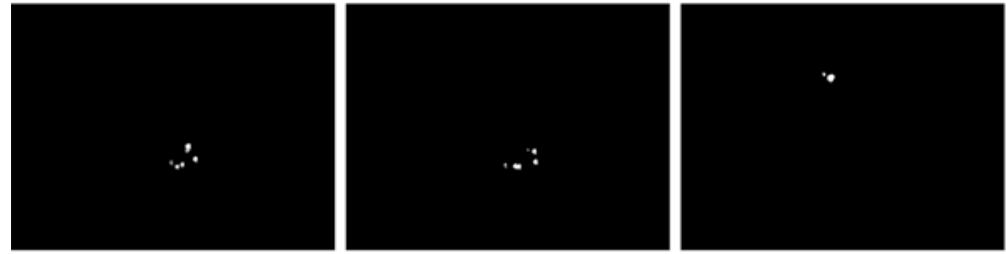

The results by our method

图 9 本文方法与信号频率模型方法的多放电点电晕检测结果

\section{5 讨论}

本文方法包含一个设置参数: $T$ 。图 10 显示了不 同 $T$ 情况下的归一化泊松概率映射图。当 $T=1$ 时, 泊松概率模型就等价于信号频率模型, 在概率映射图 中只反映每个像素出现信号点的频率。随着 $T$ 逐步增 加, 从图中可以看出, 泊松概率映射图中所检测出目 标的面积大小与强度也随之增大, 同时无效的信号点 (可认为是噪声) 也越来越明显。因此参数 $T$ 对紫外 电晕目标检测的影响, 类似于 CCD 或 CMOS 图像传 感器中利用积分时间来控制电荷累积时间长短的效 果, 这也是将参数 $T$ 称为积分时间的原因。

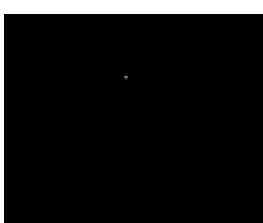

$T=1$

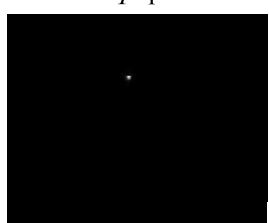

$T=20$

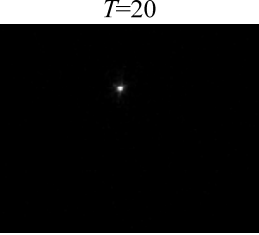

$T=100$

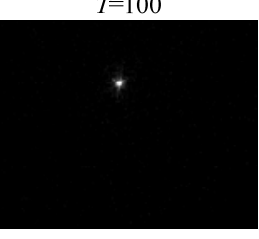

$T=200$

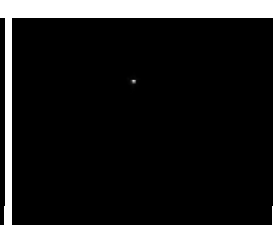

$T=10$

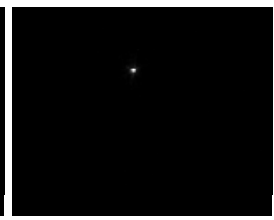

$T=50$

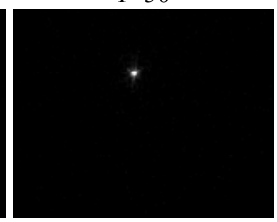

$T=150$

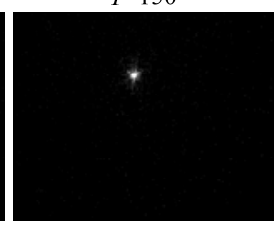

$T=300$
图 10 同积分时间 $T$ 的归一化泊松概率映射图

Fig. 10 The normalized Poisson distribution maps with various parameters $T$
积分时间 $T$ 不仅影响目标检测的效果, 同时也决 定了目标检测的更新频率。 $T$ 取值太小，则目标不明 显, 且容易丢失目标; $T$ 取值太大, 则噪声变大, 且 目标检测的更新频率降低, 不利于目标检测。通过多 次实验, 确定了 $T$ 的取值为 20 , 在本文所有试验过程 中均没有发生改变。

本文实验计算机配置为: Intel E5-1620-2.8 GHz 处理器, 4 G 内存。在 VS2012 上利用 C 语言进行编 程。经测试可知, 计算一次泊松概率映射图并将目标 提取的平均时间为 $214.2 \mathrm{~ms}$ 。

\section{4 结论}

本文提出了一种利用泊松分布的日盲紫外电晕 目标检测方法。利用紫外信号光电探测原理, 设计了 一种基于泊松分布的紫外电晕目标检测模型, 可以准 确反映探测过程中紫外电晕目标的时空域特征。针对 紫外序列图像, 首先利用暗噪声统计模型进行有无信 号的判断, 然后利用所建立的紫外电晕目标检测模型 得到紫外图像的泊松概率映射图, 最后利用改进的 Otsu 法来完成紫外电晕检测与目标提取。利用多组紫 外图像序列对本文方法进行了测试与对比，实验证明 该方法可以准确地检测出紫外图像中电晕目标, 检测 精度高, 一致性好, 可以推进紫外电晕探测在机器视 觉方面的应用。

\section{参考文献:}

[1] 匡蕾, 顾燕. 紫外像增强器用于电力安全检测的研究[J]. 红外技术, 2015, 37(11): 986-990.

KUANG Lei, GU Yan. Research on the application of UV image intensifier in security detection of power equipment [J]. Infrared Technology, 2015, 37(11): 986-990.

[2] 夏云峰, 宋新明, 戚金风. 输电线路电晕放电的天线检测方法 [J]. 中 
国电力, 2018, 51(9): 88-92.

XIA Yunfeng, SONG Xinming, QI Jinfeng, et al. Antenna detection method for corona discharge of transmission lines[J]. Electric Power, 2018, 51(9): 88-92.

[3] 王影, 王璐子, 倪进园, 等. 日盲紫外波段电晕成像特性的仿真研究 [J]. 红外技术, 2019, 41(11): 1057-1064.

WANG Hao, WANG Luzi, NI Jinyuan, et al. Research on simulation of corona imaging at solar-blind ultraviolet band[J]. Infrared Technology, 2019, 41(11): 1057-1064.

[4] LI Jiaqi, ZHOU Yue, YI Wiangyu, et al. An automatic corona-discharge detection system for railways based on solar-blind ultraviolet detection[J]. Current Optics and Photonics, 2017, 1(3): 196-202.

[5] 张海峰, 庞其昌, 陈秀春. 高压电晕放电特征及其检测 [J]. 电测与仪 表, 2006, 43(482): 6-9.

ZHANG Haifeng, PANG Qichang, CHEN Xiuchun. The characteristics of high-voltage corona and its detection[J]. Electrical Measurement\& Instrumentation, 2006, 43(482): 6-9.

[6] YANG Feng, GU Yan, SUN Jianning, et al. Research on dual spectrum solar-blind ultraviolet corona detection system [C]//2nd International Se- minar on High-Power Laser Interaction with Matter and Application, 2015: 9522.

[7] ZHANG Zhijing, ZHANG Wei, ZHANG Dongdong. Comparison of different characteristic parameters acquired by UV imager in detecting corona discharge [J]. IEEE Trans. Dielectr. Electr. Insul., 2016, 23(3): 1597-1604.

[8] 王胜辉, 律方成, 刘云鹏. 复合绝缘子电晕放电紫外图像参数的变化 特性及放电量估计 [J]. 中国电机工程学报, 2013, 33(34): 233-240.

WANG Shenghui, LÜ Fangcheng, LIU Yunpeng. Variation characteristic of composite insulator corona discharge ultraviolet image parameter and estimation of discharge magnitude[J]. Chin. Soc. for Elec. Eng., 2013, 33(34): 233-240.

[9] 周影，袁洪伟，周跃，等. 微弱日盲紫外电晕自动实时检测方法[J]. 中 国光学, 2015, 8(6): 926-932.

ZHOU Ying, YUAN Hongwei, ZHOU Yue, et al. Automatic real-time detection method of faint solar-blind ultraviolet corona[J]. Chinese Optics, 2015, 8(6): 926-932.

[10] Peter Seitz, Albert J P Theuwissen. Single Photon Imaging[M]. Heidelberg: Springer, 2011. 